\title{
EFICÁCIA E SEGURANÇA DO IMPLANTE POR CATETER DE BIOPRÓTESE VALVAR AÓRTICA (TAVR) EM PACIENTES PORTADORES DE ESTENOSE AÓRTICA
}

\author{
Rubem AB. Ramos ${ }^{1,}{ }^{*}$, Weberton D. Sobrinho², Juliana HC. de Vasconcelos ${ }^{1}$, Gabrielly FC. \\ Amorim¹, LucianoSM. Júnior¹, Antônio RC. Neto', Samuel D0. Vieira1, Nycolle B. García ${ }^{3}$, Gabriel \\ M. de Carvalho ${ }^{1}$, Fábio B. Neves ${ }^{1}$ and Dr. Evilanna L. Arruda ${ }^{4}$
}

1Faculdade de Medicina, Universidade de Rio Verde, Rio Verde, Goiás, Brasil

${ }^{2}$ Faculdade de Medicina, Universidade de Rio Verde, Campus Goianésia, Goiás, Brasil

${ }^{3}$ Faculdade de Medicina, Universidade de Grande Dourados, Mato Grosso do Sul, Brasil

${ }^{4}$ Faculdade de Farmácia, Universidade Federal de Goiás, Goiás, Brasil

\section{ARTICLE INFO}

\section{Article History:}

Received $11^{\text {th }}$ February, 2021

Received in revised form

$16^{\text {th }}$ March, 2021

Accepted $20^{\text {th }}$ April, 2021

Published online $30^{\text {th }}$ May, 2021

\section{Key Words:}

Estenose da valva aórtica; Implante de prótese valvular cardíaca;

Segurança; Eficácia.

\begin{abstract}
Introdução: O Este estudo tem como objetivo analisar, com base nos achados da literatura, a segurança e a eficácia do implante por cateter de bioprótese valvar aórtica (TAVR) com ênfase no tratamento de pacientes com estenose aórtica. Trata-se de uma revisão sistemática da literatura, de caráter qualitativo. A busca ocorreu na base de dados do MEDLINE. Os artigos foram coletados a partir de janeiro de 2010. Foram utilizados os descritores: "estenose da valva aórtica" e "implante de prótese valvular cardíaca", localizados na lista de Descritores em Ciências da Saúde (DECs). Após a aplicação dos critérios de elegibilidade, restaram 34 estudos. Pode-se concluir que o implante por cateter de bioprótese valvar aórtica (TAVR) para tratamento de pacientes com estenose aórtica apresenta diversas vantagens quando comparado ao método cirúrgico tradicional (SAVR). Ou seja, percebeu-se que a realização do TAVR é eficaz no que diz respeito aos aspectos hemodinâmicos, qualidade de vida, sobrevida, compatibilidade prótese-paciente, funcionalidade e durabilidade. Portanto, todas essas informações revelam baixos índices de disfunção valvar hemodinâmica.
\end{abstract}

*Corresponding author:

Rubem AB. Ramos

Copyright $\odot$ 2021, Rubem et al. This is an open access article distributed under the Creative Commons Attribution License, which permits unrestricted use, distribution, and reproduction in any medium, provided the original work is properly cited.

Citation: Rubem AB. Ramos, Weberton D. Sobrinho, Juliana HC. de Vasconcelos, Gabrielly FC. Amorim, LucianoSM. Júnior et al., "Eficácia e segurança do implante por cateter de bioprótese valvar aórtica (tavr) em pacientes portadores de estenose aórtica”, International Journal of Development Research, 11, (05), 47322-47328.

\section{INTRODUÇÃO}

A estenose aórtica (EA) é o estreitamento da abertura da valva aórtica que limita o fluxo de sangue do ventrículo esquerdo para a aorta por uma limitação da mobilidade da valva. É uma doença valvar comum na população ocidental, sua etiologia mais frequente é a degenerativa, seguida por reumática e congênita. Os principais fatores que envolvem a patogênese da EA degenerativa é a idade, hipertensão, turbulência do fluxo de sangue perivalvular, agregados de cálcio nas cúspides e outros processos similares ao da doença aterosclerótica envolvendo agregados lipídicos, inflamação e disfunção endotelial. (CARITÀ et al. 2016). O implante transcateter de válvula aórtica (TAVR) é um procedimento novo, no qual uma válvula bioprotética é inserida por um cateter para substituir a válvula doente, pode ser feito por via transfemoral ou apical. Ela se mostrou uma vantagem para pacientes de elevado risco cirúrgico, já que permite a inserção da válvula sem a abertura do tórax do paciente. Esse procedimento tem o potencial de prover benefícios com melhora estrutural e funcional cardíaca e se mostrou uma vantagem para a realização de troca valvar, diminuindo a morbimortalidade (GAIA et al. 2011). O TAVR é contraindicado para pacientes com expectativa de vida menor de 12 
meses e para a sua realização deve ser realizado uma angiotomografia de aorta para analisar o acesso, a válvula e prever complicações. (TARASOUTCHI et al. 2020). A cirurgia de troca valvar aórtica, melhor para pacientes com menos de 70 anos, com baixo risco cirúrgico. Há também a valvopatia aórtica por cateter-balão, ela é usada como ponte terapêutica para procedimentos em pacientes com instabilidade hemodinâmica. (TARASOUTCHI et al. 2020)

\section{MATERIAIS E MÉTODOS}

O estudo em questão caracteriza-se por uma revisão sistemática da literatura com abordagem qualitativa sobre segurança e eficácia do implante por cateter de bioprótese valvar aórtica em pacientes com estenose aórtica. Essa metodologia procura sintetizar resultados de estudos coerentes sobre este tema específico. Dessa forma, a produção do presente estudo segue os seguintes passos: (1) seleção do tema a ser abordado; (2) estabelecimento de critérios para exclusão e inclusão dos artigos científicos dentro das plataformas de pesquisas; (3) definição do conteúdo a ser extraído dos estudos selecionados e (4) elaboração da revisão que sintetize todo o conhecimento angariado. Nesta revisão de literatura, foram considerados apenas trabalhos originais publicados entre os anos de 2010 até 2020, por meio da plataforma eletrônica do MEDLINE, utilizando-se o cruzamento dos seguintes descritores em ciências da saúde (DeCS): "estenose da valva aórtica" e "implante de prótese valvular cardíaca". A seleção das pesquisas utilizadas foi realizada com base nos fatores de inclusão e exclusão citados adiante. Assim, os critérios de inclusão definidos foram: artigos originais, publicados nos idiomas inglês e português, que abordam o tema do implante por cateter de bioprótese valvar aórtica com ênfase no tratamento de pacientes portadores de estenose aórtica. Como critérios de exclusão foram estabelecidos: estudos com animais, artigos publicados que não continham respostas para o tema central da pesquisa, textos incompletos e estudos com mais de 10 anos de publicação. Ademais, foi utilizado a pirâmide de nível de evidência nas ciências médicas como ferramenta para seleção dos estudos com níveis elevados de qualidade. Posteriormente, foi construído um instrumento para integração dos achados em forma de quadro de modo a dar visibilidade às principais características de cada produção (autor, população, faixa etária, dados obtidos e discussão), mantendo-se a autenticidade das ideias, conceito e definições dos autores.

\section{RESULTADOS}

Considerando a busca dos artigos, pelo uso dos descritores, pesquisados na plataforma eletrônica MEDLINE, foi possível encontrar um total de 553 estudos. Cruzando os assuntos tratados em cada artigo haviam 295 estudos que falavam sobre "Substituição da Valva Aórtica Transcateter" e 87 artigos que tratavam sobre "bioprótese", obtendo um total de 348 artigos. Considerando o objetivo do trabalho de interrelacionar os assuntos, selecionou-se apenas os artigos que apresentavam todos os assuntos em comum. Assim sendo, após etapas da seleção final dos artigos, foram incluídos na revisão 34 artigos que atenderam aos critérios de inclusão/exclusão previamente estabelecidos e que apresentaram uma resposta a questão norteadora, sendo eles totalmente avaliados.

\section{DISCUSSÃO}

Transplantes Valvares Aórticos: A introdução da substituição transcateter de válvula aórtica (TAVR) para pacientes com estenose aórtica se apresentou como uma opção de tratamento segura para pacientes de alto e extremo risco (ADAMS et al. 2014). Nesse sentido, muitos estudos se dedicaram a comparar os procedimentos TAVR e SAVR - substituição cirúrgica da válvula aórtica.
Estudos produzidos por POPMA et al. (2019) mostraram que a substituição transcateter de válvula aórtica com o uso de uma bioprótese supraanular autoexpansível é superior à terapia médica ou cirurgia em pacientes com estenose aórtica grave que são de alto risco para complicações ou morte na cirurgia. Porém, tais estudos mostraram que a realização de TAVR em pacientes com baixo risco cirúrgico careciam de evidências de segurança e eficácia, devido à baixa mortalidade e incidência de AVC em pacientes jovens e saudáveis submetidos à SAVR.

Avaliação Gradiente Médio: Um dos parâmetros importantes avaliados no contexto cardiológico é o gradiente médio que, em casos de estenose aórtica grave, apresenta valores superiores a $50 \mathrm{mmHg}$. Dessa forma, avaliar qual o procedimento que resultará em maior melhora desses valores é imprescindível para a escolha médica, e os estudos demonstram superioridade do método TAVR em relação ao SAVR (Quadro 1). Os gradientes médios da válvula aórtica foram menores em 12 meses no grupo TAVR do que no grupo de cirurgia de acordo com os estudos de POPMA et al. (2019), que avaliou um total de 1403 pacientes dos quais 725 foram submetidos a TAVR e 678 à SAVR. Nesse mesmo sentido, um estudo realizado por MIEGHEM et al. (2019) verificou que houve diminuição do gradiente médio da válvula: de 38,0 $\pm 15,5 \mathrm{mmHg}$ para $10,8 \pm 4,6 \mathrm{~mm}$ nos pacientes submetidos à TAVR com válvula Lotus. Este mesmo estudo ainda demonstrou significativa diminuição do gradiente médio em pacientes que receberam a válvula Lotus com Depth Guard. Neste grupo a diminuição do gradiente médio da válvula foi de $39,8 \pm 13,7 \mathrm{mmHg}$ para 11,8 \pm 4,4 mmHg (MIEGHEM et al. 2019).Um ensaio clínico, realizado por SONDERGAARD et al. (2019), demonstrou que menores valores de gradiente médio ocorrem em pacientes submetidos à TAVR, $9,9 \mathrm{mmHg}$, quando comparado com os submetidos à SAVR, $14,7 \mathrm{mmHg}$. Dessa forma, demais trabalho convergiram no fato de que pacientes submetidos à TAVR apresentam grande diminuição do gradiente médio, valores estes que são menores do que os valores obtidos pelos pacientes submetidos à SAVR.

Avaliação Acidente Vascular Cerebral (AVC): Outro fator de impacto dentro dos transplantes valvares é o Acidente Vascular Cerebral (AVC). Este evento está associado ao aumento da mortalidade pós-SAVR e TAVR. A ocorrência de qualquer AVC após SAVR se associou à menor qualidade de vida, o que não aconteceu após TF-TAVR. Dentro dos estudos analisados foi obtido que a probabilidade da ocorrência de AVC foi menor entre os grupos submetidos à TAVR quando comparado com os grupos submetidos a SAVR nos 7 primeiros dias, no primeiro mês, em 1 e em 4 anos após o procedimento (KAPADIA SR et al., 2018)(Quadro 2). Há dados que não identificaram diferenças estatisticamente significativas em casos de AVC em tempo menor que 30 dias, porém demonstram menor chance na população submetida a TAVR quando comparado com a submetida a SAVR. Assim sendo, o método TAVR apresentou melhor segurança considerando o evento AVC quando comparado com SAVR. Esta afirmação também é corroborada pelo estudo PARTNER 3, onde embora os resultados cirúrgicos tenham sido excelentes: no grupo de cirurgia, a taxa de morte em 30 dias foi de $1,1 \%$, e a taxa de um composto de morte ou acidente vascular cerebral incapacitante em 1 ano foi de $2,9 \%$, o grupo TAVR apresentou melhores resultados, com taxa de morte em 30 dias foi ainda menor $(0,4 \%)$, e a taxa de morte ou AVC incapacitante em 1 ano foi de apenas 1,0\% (MACK MJ et al., 2019).

Incompatibilidade Prótese-Paciente: A incompatibilidade entre prótese e paciente ocorre quando a área efetiva do orifício de uma válvula protética implantada é muito pequena em relação ao tamanho do corpo, e demonstrou ser um forte preditor de mortalidade e degeneração bioprotética prematura em curto e longo prazo.Esse fator, que apresenta um impacto negativo na sobrevida do paciente assim como no sucesso do transplante, foi consideravelmente maior nos pacientes submetidos à SAVR, sendo que no grupo dos pacientes submetidos à TAVR a maioria dos pacientes ou não apresentaram incompatibilidade, ou apresentaram incompatibilidade leve (Quadro $3)$. 
Quadro 1 - Análise do Gradiente Médio

\begin{tabular}{|c|c|c|c|c|}
\hline Autor & População & Faixa etária & Dados obtidos & Discussão \\
\hline POPMA et al. (2019) & $\begin{array}{l}\text { Submetidos à TAVR }= \\
725 \text { Submetidos à } \\
\text { cirurgia }=678\end{array}$ & $=74$ anos & $\begin{array}{l}\text { Os gradientes médios da válvula aórtica } \\
\text { foram menores em } 12 \text { meses no grupo } \\
\text { TAVR do que no grupo de cirurgia }\end{array}$ & $\begin{array}{l}\text { Gradientes da válvula aórtica mais baixos } \\
\text { no grupo TAVR. }\end{array}$ \\
\hline $\begin{array}{l}\text { SONDERGAARD et } \\
(2019)\end{array}$ & $\begin{array}{l}\text { (A) Grupo TAVR }=139 \\
\text { (B) Grupo SAVR=135 }\end{array}$ & $\begin{array}{l}\text { (A) } 79,4 \pm 4,9 \\
\text { (B) } 78,8 \pm 4,6\end{array}$ & $\begin{array}{l}\text { Gradiente médio aórtico = 9,9 } \mathrm{mmHg} \\
\text { para TAVR e } 14,7 \mathrm{mmHg} \text { para SAVR. }\end{array}$ & $\begin{array}{l}\text { Encontra-se deterioração da válvula } \\
\text { estrutural mais grave para SAVR, } \\
\text { principalmente relacionada a gradientes } \\
\text { médios aórticos mais elevados. }\end{array}$ \\
\hline GARCÍA et al. (2018) & $\mathrm{n}=448$ pacientes & $=79,24$ anos & $\begin{array}{l}\text { O gradiente médio na alta, } 6 \text { meses e } 1 \\
\text { ano foi } 12,94( \pm 5,3) \mathrm{mmHg}, 12,19 \\
( \pm 4,7) \mathrm{mmHg} \text { e } 11,77( \pm 4,7) \mathrm{mmHg} \\
\text { respectivamente }\end{array}$ & $\begin{array}{l}\text { O gradiente médio transvalvar voltou aos } \\
\text { valores abaixo dos obtidos em estenose }\end{array}$ \\
\hline HEIMANSOHN et al. (2015) & $\begin{array}{l}\mathrm{n}=189 \text { pacientes } \\
\text { submetidos à TAVR }\end{array}$ & $=74,7 \pm 7,5$ anos & $\begin{array}{l}\text { O acompanhamento de um ano de todos } \\
\text { os } 189 \text { pacientes demonstrou gradientes } \\
\text { médios para os tamanhos das válvulas } \\
19,21,23,25 \text { e } 27 \text { mm de } 11,7,7,8,6,3, \\
4,6 \text { e } 5,0 \mathrm{mmHg} \text {, respectivamente. }\end{array}$ & $\begin{array}{l}\text { Nota-se a significativa redução dos } \\
\text { gradientes médios para todos os tamanhos } \\
\text { de válvulas, sendo que as melhores taxas } \\
\text { foram para as maiores válvulas. }\end{array}$ \\
\hline
\end{tabular}

Quadro 2. Avaliação Acidente Vascular Cerebral

\begin{tabular}{|c|c|c|c|c|}
\hline Autor & População & Faixa etária & Dados Obtidos & Discussão \\
\hline POPMA et al. (2019) & $\begin{array}{l}\mathrm{n} \text { total }=1.403 \\
\text { Submetidos à TAVR }=725 \\
\text { Submetidos à cirurgia }=678\end{array}$ & $=74$ anos & $\begin{array}{l}\text { Incidência de morte ou } \\
\text { AVC incapacitante em } \\
24 \text { meses }=5,3 \%\end{array}$ & $\begin{array}{l}\text { A TAVR com bioprótese } \\
\text { supraanular autoexpansível foi } \\
\text { associada a menor incidência de } \\
\text { AVC incapacitante. }\end{array}$ \\
\hline KAPADIA et al. (2018) & $\begin{array}{l}\text { (A) Grupo TF-TAVR } \\
\mathrm{n}=1204 \\
\text { (B) Grupo SAVR } \\
\mathrm{n}=1204\end{array}$ & $\begin{array}{l}\text { (A) } \\
=82 \pm 7,9 \\
(\mathrm{~B}) \\
=82 \pm 6,7\end{array}$ & $\begin{array}{l}\text { AVC em } 30 \text { dias = } \\
\text { SAVR }(5,1 \%) \text { e TF- } \\
\text { TAVR }(3,7 \%) . \\
\text { AVC depois de } 30 \text { dias = } \\
\text { SAVR }(3,9 \%) \text { e TF- } \\
\text { TAVR }(2,2 \%) . \\
\text { AVC em } 1 \text { ano = SAVR } \\
(6,4 \%) \text { e TF-TAVR } \\
(5,7 \%) .\end{array}$ & $\begin{array}{l}\text { Observou-se que a realização do TF- } \\
\text { TAVR está associada a uma redução } \\
\text { de } 1,7 \% \text { do risco absoluto de AVC } \\
\text { maior quando comparada com o } \\
\text { tratamento com SAVR em pacientes } \\
\text { de risco semelhante. }\end{array}$ \\
\hline MIEGHEM et al. (2019) & $\begin{array}{l}\text { A)V. Lotus: } 996 \\
\text { B)V. Lotus com Depth Guard = } \\
50\end{array}$ & $\begin{array}{l}\text { (A) } 80,8 \pm 6,5 \\
\text { (B) } \\
=80,9 \pm 8,2\end{array}$ & $\begin{array}{l}\text { (A) Taxa de AVC } \\
\text { incapacitante }=4,1 \% . \\
\text { (B) Taxa de AVC } \\
\text { incapacitante }=2 \%\end{array}$ & $\begin{array}{l}\text { Ambas as válvulas apresentam taxas } \\
\text { baixas de AVC, sendo que o } \\
\text { destaque foi para a válvula Lotus } \\
\text { com Depth Guard com probabilidade } \\
\text { bem menor de risco de AVC. }\end{array}$ \\
\hline KAPADIA et al. - (2015) & $\begin{array}{l}\mathrm{n}=1204 \text { pacientes submetidos a } \\
\text { TF- TAVR. } \\
n=1204 \text { pacientes submetidos a } \\
\text { SAVR. }\end{array}$ & $\begin{array}{l}\text { (A) SAVR: } \\
82 \pm 6,7 \text { anos } \\
\text { (B) TF-TAVR: } \\
82 \pm 7,9 \text { anos }\end{array}$ & $\begin{array}{l}\text { O AVC em } 30 \text { dias } \\
(5,1 \% \text { vs. } 3,7 \% ; p= \\
0,09) \text { foi semelhante; } \\
\text { AVC maior em } 30 \text { dias } \\
(3,9 \% \text { vs. } 2,2 \% ; \mathrm{p}= \\
0,018) \text { foi menor após } \\
\text { TF-TAVR do que } \\
\text { SAVR; }\end{array}$ & $\begin{array}{l}\text { A ocorrência de AVC em } 30 \text { dias foi } \\
\text { maior após SAVR do que após TF- } \\
\text { TAVR (3,9\% vs. } 2,2 \% \text {; }=0,018) \text {. } \\
\text { A probabilidade de AVC em } 7 \text { e } 30 \\
\text { dias, } 1 \text { e } 4 \text { anos foi de } 4,5 \%, 5,1 \% \text {, } \\
6,4 \% \text { e } 10 \% \text { após SAVR e } 3,4 \% \text {, } \\
3,7 \%, 5,7 \% \text { e } 9,3 \% \text { após TF -TAVR. }\end{array}$ \\
\hline MIEGHEM et al. - (2019) & $\mathrm{n}=1.014$ pacientes & $\begin{array}{l}80.8 \pm 6.5 \text { anos } \\
\text { Coorte: } 80.9 \pm 8.2 \\
\text { anos }\end{array}$ & $\begin{array}{lr}4,1 \% \text { dos } & \text { pacientes } \\
\text { tiveram um } & \text { acidente } \\
\text { vascular } & \text { cerebral } \\
\text { incapacitante. } & \end{array}$ & $\begin{array}{l}\text { Dados demonstram baixa } \\
\text { probabilidade da ocorrência de AVC } \\
\text { considerando o fato de se tratar de } \\
\text { uma população de idade avançada. }\end{array}$ \\
\hline
\end{tabular}

Quadro 3- Avaliação da Incompatibilidade Prótese-Paciente

\begin{tabular}{|c|c|c|c|c|}
\hline Autor & População & Faixa etária & Dados Obtidos & Discussão \\
\hline $\begin{array}{l}\text { THYREGOD et al. } \\
-(2016)\end{array}$ & $\mathrm{n}=280$ pacientes & $\geq 70$ anos & $\begin{array}{llll}\text { IPP nenhuma/leve: } & \text { TAVR }=59,4 \% \text { vs } \\
\text { SAVR }=29,40 \% ; & \text {-IPP } & \text { moderada: } \\
\text { TAVR }=35,5 \% \text { vs SAVR }=36,70 \% & \\
\text {-IPP grave: } & \text { TAVR }=14 \% & \text { vs } \\
\text { SAVR }=33,90 \% . & & & \end{array}$ & $\begin{array}{l}\text { A incompatibilidade prótese-paciente grave } \\
\text { ou moderada foi significativamente maior } \\
\text { no grupo SAVR. }\end{array}$ \\
\hline $\begin{array}{l}\text { ZORN et al. } \\
(2015)\end{array}$ & $\begin{array}{l}\mathrm{n}=742 \text { pacientes } \\
\text { submetidos à TAVR } \\
\text { ou à SAVR }\end{array}$ & $\bar{x}=83,2$ anos & 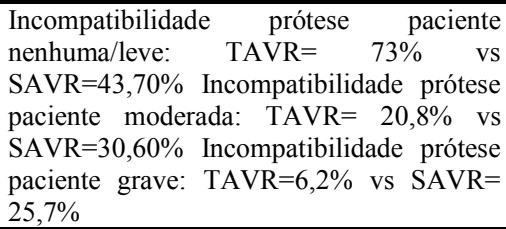 & $\begin{array}{l}\text { O presente estudo demonstra superioridade } \\
\text { da segurança e eficácia do transplante } \\
\text { transcateter da bioprótese em relação aos } \\
\text { pacientes submetidos à SAVR }\end{array}$ \\
\hline $\begin{array}{l}\text { POPMA et al. - } \\
(2019)\end{array}$ & $\begin{array}{l}\mathrm{n} \text { total }=1.403 \\
\text { Submetidos à } \\
\text { TAVR }=725 \\
\text { Submetidos à } \\
\text { cirurgia }=678\end{array}$ & $=74$ anos & $\begin{array}{l}\text { A incompatibilidade paciente-prótese grave } \\
\text { ocorreu aos } 12 \text { meses em } 1,8 \% \text { dos } \\
\text { pacientes submetidos à TAVR e } 8,2 \% \text { nos } \\
\text { pacientes submetidos à SAVR }\end{array}$ & $\begin{array}{l}\text { TAVR apresentou melhores resultados } \\
\text { quando avaliada a incompatibilidade prótese } \\
\text { paciente. }\end{array}$ \\
\hline
\end{tabular}


Quadro 4. Avaliação da Ocorrência de Regurgitação

\begin{tabular}{|c|c|c|c|c|}
\hline Artigo & População & Faixa etária & Dados Obtidos & Discussão \\
\hline $\begin{array}{l}\text { Mieghen et al } \\
\text { (2019) }\end{array}$ & $\mathrm{n}=1.014$ pacientes & $\begin{array}{c}80.8 \pm 6.5 \text { anos } \\
\text { Coorte: } 80.9 \pm 8.2 \\
\text { anos }\end{array}$ & $\begin{array}{l}\text { Vazamento paravalvar estava ausente ou } \\
\text { traçado em } 94,5 \% \text {, leve em } 5,1 \% \mathrm{e} \\
\text { moderado em } 0,4 \% \text { dos pacientes. }\end{array}$ & $\begin{array}{c}\text { Os pacientes submetidos à } \\
\text { TAVR apresentam baixa } \\
\text { probabilidade de apresentarem } \\
\text { vazamento paravalvar } \\
\end{array}$ \\
\hline $\begin{array}{l}\text { García et al } \\
\text { (2018) }\end{array}$ & $\mathrm{n}=448$ pacientes & $79,24 \pm 4.1$ anos & $\begin{array}{l}\text { Na alta, } 12(2,6 \%) \text { pacientes apresentavam } \\
\text { vazamento periprotético mínimo e } 4 \\
(0,89 \%) \text { pacientes apresentavam } \\
\text { vazamento moderado. Houve } 3 \\
\text { reintervenções durante o acompanhamento } \\
\text { (2 endocardite e } 1 \text { disfunção devido à } \\
\text { progressão do vazamento periprotético). }\end{array}$ & $\begin{array}{l}\text { Um transplante realizado por um } \\
\text { meio menos invasivo parece } \\
\text { contribuir para uma menor } \\
\text { chance de vazamento, assim } \\
\text { como a qualidade da válvula } \\
\text { bioprotética implantada }\end{array}$ \\
\hline $\begin{array}{c}\text { MONTONE et } \\
\text { al. - (2017) }\end{array}$ & $\mathrm{n}=225$ pacientes & $=82,6 \pm 6,3$ anos & $\begin{array}{c}\text { A regurgitação paravalvar (RVP) era traço } \\
\text { / leve em } 99,1 \% \text { dos pacientes na alta. } \\
\text { Apenas dois pacientes }(0,9 \%) \text { tiveram RVP } \\
\text { moderada na alta e em } 30 \text { dias, enquanto } \\
\text { nenhum teve RVP grave. }\end{array}$ & $\begin{array}{c}\text { A regurgitação apresentou } \\
\text { pequena incidência no grupo de } \\
\text { pacientes submetidos a TAVR } \\
\text { analisados. }\end{array}$ \\
\hline
\end{tabular}

Quadro 5. Avaliação da Disfunção de válvula estrutural (SVD) e não estrutural (NSVD)

\begin{tabular}{|c|c|c|c|c|}
\hline Artigo & População & \begin{tabular}{|l|} 
Fa1xa etárıa \\
\end{tabular} & \begin{tabular}{|c|} 
Dados Obtidos \\
\end{tabular} & Discussão \\
\hline $\begin{array}{l}\text { SONDERGAARD } \\
\text { et al. - (2019) }\end{array}$ & $\begin{array}{lll}\text { (A) } & \text { Grupo } & \text { TAVR }= \\
139 & & \\
\text { (B) } & \text { Grupo } & \text { SAVR }= \\
135 & & \\
\end{array}$ & $\begin{array}{l}(\mathrm{A}) 79,4 \pm 4,9 \\
\text { anos } \\
\text { (B) 78,8 } \pm 4,6 \\
\text { anos }\end{array}$ & $\begin{array}{l}\text { Deterioração da válvula estrutural } \\
\text { (SVD): } 24 \% \text { para SAVR e } 4,8 \% \text { para } \\
\text { TAVR. }\end{array}$ & $\begin{array}{l}\text { SAVR foi a opção que culminou em maior } \\
\text { probabilidade da ocorrência de SVD. }\end{array}$ \\
\hline $\begin{array}{l}\text { GERCKENS et al. } \\
-(2017)\end{array}$ & $\begin{array}{l}\mathrm{n} \text { inicial }=996 \mathrm{n} \text { de } 5 \\
\operatorname{anos}=465\end{array}$ & $=81$ anos & $\begin{array}{l}\text { Deterioração da válvula estrutural em } 1 \\
\text { ano }=0,2 \% \\
\text { Deterioração da válvula estrutural em } 5 \\
\text { anos }=0,9 \%\end{array}$ & $\begin{array}{l}\text { As taxas de deterioração analisadas nos } \\
\text { procedimentos transcateter possuíram baixos } \\
\text { valores. }\end{array}$ \\
\hline
\end{tabular}

Quadro 6. Avaliação da Mortalidade e Sobrevida

\begin{tabular}{|c|c|c|c|c|}
\hline Artigo & População & Faixa etária & Dados Obtidos & Discussão \\
\hline $\begin{array}{l}\text { MIEGHEM et al. } \\
(2019)\end{array}$ & $\begin{array}{l}\text { A)Válvula Lotus: } \\
\text {-Submetidos à TAVR=996 } \\
\text { B) Válvula Lotus com } \\
\text { Depth Guard: } \\
\text {-Submetidos à TAVR=5 }\end{array}$ & $\begin{array}{l}\text { (A) } \\
=80,8 \pm 6,5 \\
\text { (B) } \\
=80,9 \pm 8,2\end{array}$ & $\begin{array}{l}\text { (A) Incidência de morte por todas as } \\
\text { causas em } 1 \text { ano }=11,7 \% \\
\text { Incidência de mortalidade } \\
\text { cardiovascular }=7,6 \% \\
\text { (B) Incidência de morte por todas as } \\
\text { causas }=0\end{array}$ & $\begin{array}{l}\text { A Válvula Lotus com Depth Guard mostrou } \\
\text { taxas de mortalidade baixas demonstrando } \\
\text { que o transplante pela via transcateter } \\
\text { utilizando a válvula citada são seguros e } \\
\text { eficazes }\end{array}$ \\
\hline $\begin{array}{l}\text { TARANTINI et al. } \\
-(2019)\end{array}$ & $\begin{array}{l}\text { A) G. baixo risco }=518 \\
\text { B) G. risco intermediário }= \\
691 \text { C) G. de risco grave }= \\
576 \text { Submetidos à TAVR: } \\
\cong 1 / 2 \text { cada grupo }\end{array}$ & $\begin{array}{l}\text { A) }=78,4 \pm \\
7,44 \\
B)=82,9 \pm \\
5,67 \\
\text { C) }=83,0 \pm \\
5,71\end{array}$ & $\begin{array}{l}\text { Taxa de mortalidade por todas as } \\
\text { causas em } 1 \text { ano }=(\mathrm{A})-10,3 \%,(\mathrm{~B})- \\
11,4 \%,(\mathrm{C})-17,1 \% \text { Incidência de } \\
\text { mortalidade cardiovascular em } 1 \text { ano }= \\
(\mathrm{A})-5,3 \%,(\mathrm{~B})-7,7 \%,(\mathrm{C})-11,4 \%\end{array}$ & $\begin{array}{l}\text { A mortalidade mostrou-se crescente } \\
\text { conforme um grau de risco mais elevado, } \\
\text { porém os valores entre baixo risco e risco } \\
\text { grave não foram tão diferentes nos pacientes } \\
\text { submetidos a TAVR, indicando melhor uso } \\
\text { por pacientes de alto risco. }\end{array}$ \\
\hline $\begin{array}{l}\text { MOLLMANN et } \\
\text { al. - (2017) }\end{array}$ & $\begin{array}{l}\mathrm{n} \text { inicial }=222 \mathrm{n} \text { de } \\
\text { implantados por TAVR }= \\
220\end{array}$ & $83,0 \pm 4,6$ anos & $\begin{array}{l}\text { Mortalidade imediata do procedimento } \\
=1,4 \% \text { Mortalidade por todas as } \\
\text { causas em } 30 \text { dias }=3,6 \%\end{array}$ & $\begin{array}{l}\text { Nota-se baixa mortalidade mesmo o } \\
\text { procedimento ocorrendo em pacientes com } \\
\text { idade elevada, média de } 83 \text { anos. }\end{array}$ \\
\hline $\begin{array}{l}\text { POPMA et al. - } \\
(2017)\end{array}$ & $\begin{array}{l}\text { Submetidos à TAVR } \\
\mathrm{n}=241\end{array}$ & $\begin{array}{l}=83,3 \pm 7,2 \\
\text { anos }\end{array}$ & $\begin{array}{l}\text { Mortalidade por todas as causas }= \\
2,5 \% . \text { Todas foram de natureza } \mathrm{CV} \text {. }\end{array}$ & $\begin{array}{l}\text { Baixa mortalidade em população idosa, } \\
\text { sendo todas elas relacionadas a eventos } \\
\text { cardiovasculares. }\end{array}$ \\
\hline
\end{tabular}

Quadro 7. Avaliação da Área Efetiva do Orifício

\begin{tabular}{|c|c|c|c|c|}
\hline Artigo & Populaçáo & Faixa etária & Dados Obtidos & Discussão \\
\hline POPMA et al. - (2019) & $\begin{array}{l}\text { Submetidos à } \mathrm{TAVR}=725 \\
\text { Submetidos à cirurgia }=678\end{array}$ & $=74$ anos & EOA $>$ para TAVR do que SAVR. & $\begin{array}{l}\text { Condizente com os baixos valores de } \\
\text { gradiente médio, o que destaca uma } \\
\text { boa avaliação do procedimento }\end{array}$ \\
\hline $\begin{array}{l}\text { SONDERGAAD et al. - } \\
(2019)\end{array}$ & $\begin{array}{l}\text { (A) Grupo TAVR }=139 \\
\text { (B) Grupo SAVR }=135\end{array}$ & $\begin{array}{l}(\mathrm{A}) 79,4 \pm 4,9 \\
\text { anos } \\
(\mathrm{B}) 78,8 \pm 4,6 \\
\text { anos }\end{array}$ & $\begin{array}{l}\text { EOA (Área Efetiva do Orifício) }= \\
\text { maior para TAVR do que SAVR } \\
\left(1,53 \mathrm{~cm}^{2} \text { vs } 1,16 \mathrm{~cm}^{2}\right) .\end{array}$ & $\begin{array}{l}\text { Superioridade do método } \\
\text { quando comparado com o método } \\
\text { SAVR, tendo em vista os maiores } \\
\text { valores de EOA. }\end{array}$ \\
\hline $\begin{array}{l}\text { MOLLMANN et al.- } \\
(2013)\end{array}$ & $\mathrm{n}=20$ pacientes & $\begin{array}{l}=84,8 \pm 4,5 \\
\text { anos }\end{array}$ & $\begin{array}{l}\text { EOA melhorou de } 0,7 \mathrm{~cm}^{2} \text { para } 1,8 \\
\mathrm{~cm}^{2} \text {. }\end{array}$ & $\begin{array}{l}\text { Eficiência clara do método TAVR } \\
\text { quando comparada a área efetiva do } \\
\text { orifício }\end{array}$ \\
\hline $\begin{array}{l}\text { GERCKENS et al. - } \\
(2017)\end{array}$ & $\begin{array}{l}\mathrm{n} \text { inicial }=996 \\
\mathrm{n} \text { de } 5 \text { anos }=465\end{array}$ & $=81$ anos & $\begin{array}{l}\text { EOA }=\text { melhorou de } 0,8 \pm 0,5 \mathrm{~cm}^{2} \\
\text { no início do estudo para } 1,7 \pm 0,4 \\
\mathrm{~cm}^{2} \text { em } 5 \text { anos }\end{array}$ & $\begin{array}{l}\text { Destaca-se bons resultados com } \\
\text { elevação considerável da área efetiva } \\
\text { do orifício. }\end{array}$ \\
\hline $\begin{array}{l}\text { MOLLMANN et al. } \\
(2017)\end{array}$ & $\begin{array}{l}\mathrm{n} \text { inicial }=222 \\
\text { número de implantados por } \\
\text { TAVR }=220\end{array}$ & $\begin{array}{l}=83,0 \pm 4,6 \\
\text { anos }\end{array}$ & $\begin{array}{l}\text { Área da válvula }=\text { aumentou de } 0,7 \\
\pm 0,2 \text { para } 1,9 \pm 0,5 \mathrm{~cm}^{2} \\
\text { EOA }=\text { aumentou de } 0,4 \pm 0,1 \text { para } \\
1,1 \pm 0,3 \mathrm{~cm}^{2} / \mathrm{m}^{2}\end{array}$ & $\begin{array}{l}\text { O procedimento via TAVR apresenta } \\
\text { boa eficácia quando se analisa o } \\
\text { aumento considerável da EOA. }\end{array}$ \\
\hline
\end{tabular}


Um estudo realizado por Thyregod HGH, et al (2016) demonstrou uma probabilidade cerca de duas vezes maior de incompatibilidade prótese-paciente em indivíduos submetidos à SAVR $(33,90 \%)$, quando comparado com os pacientes que foram submetidos à TAVR $(14 \%)$.

Vazamento/ Regurgitação: Ocorre geralmente por causa de uma infecção ou pela cicatrização incompleta na área que acaba culminando na ruptura dos pontos realizados durante a cirurgia. Segundo as estatísticas, o problema afeta entre $2 \%$ e $15 \%$ dos pacientes submetidos ao procedimento de substituição de válvula (Quadro 4). No entanto, o risco é maior entre aqueles que já passaram por um número maior de procedimentos cirúrgicos cardíacos. Um transplante realizado por um meio menos invasivo parece contribuir para uma menor chance de vazamento, assim como a qualidade da válvula bioprotética implantada (GARCÍA EB et al., 2018). Nesse sentido os estudos demonstram boa segurança e eficácia do método TAVR, uma vez que todos os estudos analisados apontaram que os pacientes submetidos ao transplante transcateter apresentaram pequena probabilidade de apresentarem vazamento paravalvar. $\mathrm{O}$ estudo de Montone et al (2017) obteve que da amostra de sua pesquisa apenas dois pacientes $(0,9 \%)$ tiveram regurgitação paravalvar (RVP) moderada na alta e em 30 dias, enquanto nenhum teve RVP grave. A regurgitação paravalvar (RVP) era traço/leve em $99,1 \%$ dos pacientes na alta.

Disfunção de válvula estrutural (SVD) e não estrutural (NSVD): Sondergaard et al (2019) analisaram a durabilidade da válvula aórtica transcateter e da bioprotética cirúrgica, encontrando uma deterioração da válvula estrutural mais grave para SAVR ( $24 \%$ em relação à $4,8 \%$ para TAVR), a qual está relacionada a gradientes médios aórticos mais elevados (Quadro 5). Dentro desse contexto, é interessante ressaltar que os pacientes mais jovens correm maior risco de deterioração da válvula bioprotética pós-SAVR e necessidade de reintervenção. Embora isso possa ser realizado como um procedimento transcateter menos invasivo, a relação risco-benefício precisa ser aceitável, pois ao se expandir a terapia para pacientes mais jovens, de baixo risco e com maior expectativa de vida, a durabilidade da válvula é um fator importante a ser considerado segundo o autor. Então, para comparar as diferentes biopróteses usadas para SAVR e TAVR, definições padronizadas de disfunção e falha da válvula bioprotética precisam ser aplicadas. Nesse mesmo estudo, não houve diferença significativa na deterioração da válvula não estrutural (NSVD) entre os grupos TAVR e SAVR por 6 anos. Ademais, vale ressaltar que o estudo de GERCKENS et al. (2017) apresentou como componentes da SVD: disfunção ou deterioração da válvula experimental, exclusiva de infecção ou trombose; alterações intrínsecas à válvula, como desgaste, fratura, calcificação, ruptura do folheto, deslocamento do stent ou rompimento da linha de sutura dos componentes de uma válvula experimental. Baseando-se nesses componentes, tal estudo mostrou que os pacientes submetidos à TAVR apresentaram uma deterioração da válvula estrutural em 1 ano de $0,2 \%$ e em 5 anos de $0,9 \%$, evidenciando uma baixa taxa de disfunção da válvula estrutural (SVD) em pacientes que realizaram a substituição transcateter de válvula aórtica.

Popma et al. (2019) realizou um estudo para comparar a segurança e eficácia da TAVR usando a bioprótese autoexpansível com a SAVR em pacientes de baixo risco cirúrgico. Como resultado, obteve-se que a SVD foi mais significativa para SAVR do que para TAVR nos quesitos gradientes médios (GM) e área efetiva do orifício (EOA), pois os GM foram menores em 12 meses para TAVR e a EOA foi maior para TAVR. Em contraposição, as taxas de regurgitação aórtica foram maiores no grupo TAVR. Obteve-se, também, que a NSVD foi mais significativa para SAVR, porque a incompatibilidade pacienteprótese (IPP) grave em 12 meses ocorreu em 1,8\% dos pacientes TAVR e em $8,2 \%$ no grupo SAVR.

Mortalidade e Sobrevida: Muitos artigos verificados analisaram a incidência de mortalidade pós-TAVR como parâmetro para avaliação da qualidade de vida dos pacientes após o procedimento (Quadro 6).
Com relação às taxas de mortalidade por todas as causas, a incidência de morte por qualquer causa em 30 dias é inferior no grupo TAVR $(0,5 \%)$ quando comparado com o grupo de cirurgia $(1,3 \%)$. A incidência de mortalidade em 24 meses foi de $4,5 \%$ no grupo TAVR e $4,5 \%$ no grupo de cirurgia, concluindo que a TAVR com uma bioprótese supraanular autoexpansível para pacientes de baixo risco não é inferior à SAVR com relação ao risco de morte em 24 meses (POPMA et al., 2019).Ainda nesse contexto de análise da submissão de pacientes de baixo risco cirúrgico para a TAVR, Sondergaard et al. (2016) demonstrou em um ensaio clínico randomizado que a segurança e a eficácia do TAVR são semelhantes à segurança e à eficácia da cirurgia em pacientes de baixo risco com estenose aórtica grave sintomática.

Mieghem et al. (2019), em seu estudo prospectivo de coorte, submeteu pacientes ao procedimento TAVR com 2 tipos diferentes de válvulas (sendo a segunda semelhante a primeira, porém com modificações em seu design), apresentando uma baixa taxa de mortalidade em ambos os casos, evidenciando, portanto, a segurança e eficácia do procedimento TAVR quando se usa uma válvula eficiente. Tal estudo também apresentou a possível influência do vazamento paravalvar na mortalidade, sendo esta maior conforme mais grave é o vazamento. Vale ressaltar que, no estudo de TARANTINI et al. (2019), a incidência de mortalidade por todas as causas foi inferior em qualquer das categorias de risco em comparação com o que seria esperado com a cirurgia SAVR (3:10). Esse estudo também mostra que a idade continua sendo um dos motivos mais importantes para a decisão da equipe de optar pela TAVR, pois nos principais estudos recentes e em andamento, os pacientes submetidos à TAVR tem, predominantemente, idade $>80$ anos. O estudo de POPMA et al. (2017) também demonstrou baixos índices de mortalidade por todas as causas pós-TAVR: 2,5\% apenas. DVIR et al. (2012) analisou as taxas de mortalidade em 30 dias de pacientes submetidos à implantação transcateter de válvula aórtica em válvula (VIV), a qual é uma alternativa terapêutica emergente para pacientes com bioprótese cirúrgica com falha e que pode evitar a necessidade de reoperação, e verificou-se que a incidência foi de $8,4 \%$, sendo, então, comparável aos índices em outras coortes de TAVR. Gerckens et al. (2017) demonstrou que a taxa de mortalidade por todas as causas em 1 ano entre os pacientes submetidos à TAVR foi de $17,6 \%$ e em 5 anos foi de 50,7\%. Ele considerou que esse índice de 5 anos está dentro da faixa esperada para esta população TAVR idosa.

Área Efetiva do Orifício (EOA): Sabe-se que, após a realização da TAVR, muitos parâmetros são utilizados para acompanhamento dos pacientes submetidos à substituição transcateter a fim de se analisar a eficácia e segurança do procedimento. Um desses parâmetros é a área efetiva do orifício (EOA)(Quadro 7). Nesse aspecto, o estudo prospectivo não randomizado de Gerckens et al. (2017), o qual acompanhou 465 pacientes pós-TAVR ao longo de 5 anos, demonstrou uma relação entre gradientes de válvula aórtica (GM) mais baixos com áreas efetivas do orifício (EOA) maiores. No início do acompanhamento, o gradiente médio era de 45,3 $\pm 15,9 \mathrm{mmHg}$ em relação a uma EOA de $0,8 \pm 0,5 \mathrm{~cm}^{2}$. Após a alta dos pacientes, percebeu-se que o GM caiu para $9,8 \pm 4,4 \mathrm{mmHg}$ enquanto a EOA aumentou para $1,7 \mathrm{~cm} \pm 0,4 \mathrm{~cm}^{2}$. Portanto, notou-se que a realização da TAVR é efetiva com relação aos aspectos hemodinâmicos, pois houve baixa taxa de disfunção da válvula hemodinâmica. Seguindo esse raciocínio, POPMA et al. (2019), assim como HAHN et al. (2019) também demonstraram que gradientes da válvula aórtica mais baixos se relacionam com áreas efetivas do orifício (EOA) maiores no grupo TAVR. Semelhantemente, DALMAU et al. (2011) mostrou que gradientes transvalvares médios significativamente menores se relacionam a maiores EOAs. Então, corroborou-se a relação entre baixos gradientes médios e maiores áreas efetivas de orifício, algo que é proporcionado pela TAVR, demonstrando, assim, a eficácia do procedimento para pacientes com estenose aórtica grave. Nesse contexto, Mollmann et al. (2013) analisou que o uso de uma válvula supra-anular autoexpansível também acarretou numa melhora significativa da EOA de $0,7 \mathrm{~cm}^{2}$ para $1,8 \mathrm{~cm}^{2}$ em pacientes submetidos à TAVR. 


\section{CONCLUSÃO}

De acordo com o resultado do estudo, pode-se concluir que o implante por cateter de bioprótese valvar aórtica (TAVR) para tratar pacientes com estenose aórtica possui diversas vantagens quando comparado com o método tradicional cirúrgico (SAVR) em pacientes com alto risco de morte. Pelos dados obtidos no primeiro tópico onde se analisa o gradiente médio em pacientes submetidos à TAVR em comparação com o método SAVR, pode-se atestar a eficácia através dos dados que contemplam menores valores para pacientes submetidos ao método transcateter, principalmente aqueles que receberam a válvula LÓTUS, evidenciando assim o seu sucesso entre os pacientes de alto risco cirúrgico.Analisando a incidência de acidente vascular encefálico (AVC) pós tratamento da estenose por SAVR ou TAVR, concluiu-se que pessoas submetidas ao método cirúrgico, a longo prazo, apresentam piora em sua qualidade de vida, além de que as próprias chances de se desenvolver um acidente vascular encefálico se tornam maiores.Em relação ao terceiro tópico e a incompatibilidade prótese-paciente, capta-se maiores valores de rejeição para pacientes submetidos ao método cirúrgico, sendo que através do método TAVR há incompatibilidade leve, ou não há.Em análise do quarto tópico, a incidência de vazamento/ regurgitação em pacientes que passam por métodos menos invasivos se torna muito menor em relação a pessoas que foram submetidas a vários procedimentos, afirmando o sucesso do método transcateter.As disfunções estruturais da válvula (SVD) e um maior número de reintervenções acometeram pacientes que se submeteram ao método cirúrgico tradicional. Os dados obtidos revelam maior durabilidade e consistência estrutural através do método transcateter, porém danos não estruturais (NSVD) não apresentam diferenças entre os dois métodos.No sexto tópico entende-se através de dados que tratam a sobrevida dos pacientes que passaram pelos dois métodos, que em 30 dias, as taxas de morte por todas as causas são menores para pacientes TAVR, porém em 24 meses a mesma taxa se torna igualitária entre os dois métodos.Tal dado evidencia que o procedimento de transplante por via transcateter para pacientes de baixo risco não é inferior à SAVR com relação ao risco de morte em 24 meses. Para concluir, o sétimo e último tópico trata sobre a área efetiva do orifício (EOA), trazendo dados que correlacionam menores taxas de gradiente médio conforme aumentam-se essas áreas. Tais conclusões afirmam o triunfo do TAVR, pois em outros trabalhos pode-se afirmar que pacientes tratados por esse método possuem menores gradientes médios, e assim induz-se que a EOA dos mesmos se torna maiores. Todas essas informações revelam baixas taxas de disfunções da válvula hemodinâmica.

Agradecimentos: Aos colegas que ajudaram na realização desse projeto.

\section{REFERÊNCIAS}

Carità, P., Coppola, G., Novo, G., Caccamo, G., Guglielmo, M., Balasus, F., Novo, S., Castrovinci, S., Moscarelli, M., Fattouch, K., Corrado, E. 2016. Aortic stenosis: insights on pathogenesis and clinical implications. Disponível online em:https://www.ncbi.nlm.nih.gov/pmc/articles/PMC4987417/

Dalmau, M. J., González-Santos, J. M., Blázquez, J. A., Sastre, J. A., López-Rodríguez, J., Bueno, M., Castaño, M., \& Arribas, A. 2011. Hemodynamic performance of the Medtronic Mosaic and Perimount Magna aortic bioprostheses: five-year results of a prospectively randomized study. Disponível online em:https://academic.oup.com/ejcts/article/39/6/844/376182

Dvir, D., Webb, J., Brecker, S., Bleiziffer, S., Hildick-Smith, D., Colombo, A., Descoutures, F., Hengstenberg, C., Moat, N. E., Bekeredjian, R., Napodano, M., Testa, L., Lefevre, T., Guetta, V., Nissen, H., Hernández, J. M., Roy, D., Teles, R. C., Segev, A., Dumonteil, N., ... Kornowski, R. 2012. Transcatheter aortic valve replacement for degenerative bioprosthetic surgical valves: results from the global valve-in-valve registry. Disponível online em:https://www.ahajournals.org/doi/10.1161/CIRCULATIONAH A.112.104505
Gaia, DF., Palma, JH., Ferreira, CBND., Souza, JAM., Gimenes, MV., Macedo, MT., Martins, MR., Buffolo, E. 2011. Implante transcateter de valva aórtica: resultados atuais do desenvolvimento e implante de um nova prótese brasileira. Disponível online em: https://repositorio. unifesp.br/ handle/11600/6590

Gerckens, U., Tamburino, C., Bleiziffer, S., Bosmans, J., Wenaweser, P., Brecker, S., Guo, J., \& Linke, A. 2017. Final 5-year clinical and echocardiographic results for treatment of severe aortic stenosis with a self-expanding bioprosthesis from the ADVANCE Study. Disponível online em: https://academic.oup.com/ eurheartj/article/38/36/2729/3867643

Heimansohn, D., Roselli, E. E., Thourani, VH., Wang, S., Voisine, P., Ye, J., Moon, M. 2016. North American trial results at 1 year with the Sorin Freedom SOLO pericardial aortic valve. Disponível online em:https://academic.oup.com/ ejcts/article/49/2 /493/2 465391

Klautz, RJ., Kappetein, AP., Lange, R., Dagenais, F., Labrousse, L., Bapat, V. 2017. Safety, effectiveness and haemodynamic performance of a new stented aortic valve bioprosthesis. Disponível online em:https://academic.oup.com/ejcts/ article/52/3/ 425/3798543

Mack, MJ., Leon, MB., Thourani, VH., Makkar, R., Kodali, SK., Russo, M., Kapadia, SR., Malaisrie, SC., Cohen, DJ., Pibarot, P., Leipsic, J., Hahn, RT., ... Genereux, P.2019. Transcatheter AorticValve Replacement with a Balloon-Expandable Valve in LowRisk Patients. Disponível online em:https://www.nejm.org/ doi/10.1056/NEJMoa1814052

Möllmann, H., Diemert, P., Grube, E., Baldus, S., Kempfert, J., Abizaid, A. 2013. Symetis ACURATE TFTM aortic bioprosthesis. Disponível online em:https://eurointervention.pcronline.com/ article/symetis-acurate-tf-aortic-bioprosthesis

Möllmann H., Linke A, Holzhey DM., Walther T, Manoharan G., Schäfer U., Heinz-Kuck K., Van Boven AJ., Redwood SR., Kovac J., Butter C., Søndergaard L., Lauten A., Schymik G., Worthley SG. Implantation and 30-Day Follow-Up on All 4 Valve Sizes Within the Portico Transcatheter Aortic Bioprosthetic Family. Disponível online em:https://www.sciencedirect. com/science/article/pii/S1936879817309706?via\%3Dihub

Montone, RA., Testa, L., Fraccaro, C., Montorfano, M., Castriota, F., Nerla, R., Angelillis, M., Tusa, M., Giannini, F., Garatti, A., Tarantini, G., Petronio, AS., Brambilla, N., Bedogni, F. 2017. Procedural and 30-day clinical outcomes following transcatheter aortic valve replacement with lotus valve: Results of the RELEVANT study. Disponível online em:https://onlinelibrary.wiley.com/doi/abs/10.1002/ccd.26914

Popma, JJ., Deeb, GM., Yakubov, SJ., Mumtaz, M., Gada, H., O'Hair, D., Bajwa, T., Heiser, JC., Merhi, W., Kleiman, NS., Askew, J., Sorajja, P., Rovin, J., Chetcuti, SJ., Adams, DH., Teirstein, PS., Zorn, GL., Forrest, JK., Tchétché, D., Resar, J. Evolut Low Risk Trial Investigators 2019. Transcatheter Aortic-Valve Replacement with a Self-Expanding Valve in Low-Risk Patients. Disponível online em:https://www.nejm.org/doi/10.1056/NEJMoa1816885

Popma, J. J., Reardon, M. J., Khabbaz, K., Harrison, J. K., Hughes, G. C., Kodali, S., George, I., Deeb, G. M., Chetcuti, S., Kipperman, R., Brown, J., Qiao, H., Slater, J., \& Williams, M. R. 2017. Early Clinical Outcomes After Transcatheter Aortic Valve Replacement Using a Novel Self-Expanding Bioprosthesis in Patients With Severe Aortic Stenosis Who Are Suboptimal for Surgery: Results of the Evolut R U.S. Study. Disponível online em: https://www.sciencedirect.com/science/article/pii/S193687981631 6260?via\%3Dihub

Tarasoutchi, F., Montera, MW., Ramos, AIO., Sampaio, RO., Rosa, VEE., Accorsi, TAD., Santis, A., Fernandes, JRC., Pires, LJT., Spina, GS., Vieira, MLC., Lavitola, PL., Ávila, WS., Paixão, MR., Bignoto, T., Togna, DJD., Mesquita, ET., ... Saraiva, JFK. 2020. Disponível online em:http://abccardiol.org/en/ article/update-of-the-brazilian-guidelines-for-valvular-heartdisease-2020/

Thyregod, H. G., Steinbrüchel, D. A., Ihlemann, N., Ngo, T. A., Nissen, H., Kjeldsen, B. J., Chang, Y., Hansen, P. B., Olsen, P. S., \& Søndergaard, L. 2016. No clinical effect of prosthesis-patient 
mismatch after transcatheter versus surgical aortic valve replacement in intermediate- and low-risk patients with severe aortic valve stenosis at mid-term follow-up: an analysis from the NOTION trial. Disponível online em:https:/academic.oup.com/ ejcts/article/50/4/721/2197369
Zorn, G. L., Little, S. H., Tadros, P., Deeb, GM., Gleason, TG., Heiser, J., Kleiman, NS., Oh, JK., Popma, JJ., Adams, D., Huang, J., Reardon, MJ. 2016. Prosthesis-patient mismatch in high-risk patients with severe aortic stenosis: A randomized trial of a selfexpanding prosthesis. Disponível online em:https://www.jtcvs .org/article/S0022-52231502133-9/fulltext 